\title{
Longitudinal field study on bovine Babesia spp. and Anaplasma phagocytophilum infections during a grazing season in Belgium
}

\author{
Laetitia Lempereur • Maude Lebrun • \\ Pascale Cuvelier • Géraldine Sépult • Yannick Caron • \\ Claude Saegerman • Brian Shiels $\cdot$ Bertrand Losson
}

Received: 2 August 2011 / Accepted: 13 September 2011 / Published online: 27 September 2011

(C) Springer-Verlag 2011

\begin{abstract}
Anaplasmosis and babesiosis are major tick-borne diseases with a high economic impact but are also a public health concern. Blood samples collected in the spring, summer, and autumn of 2010 from 65 cows in seven different farms in Belgium were monitored with an indirect immunofluorescence antibody test to assess seroprevalence against these pathogens. Seroprevalences to Babesia spp. were measured as $10.7 \%$, $20 \%$, and $12.3 \%$ in spring, summer, and autumn, respectively, whereas seroprevalences to Anaplasma phagocytophilum were $30.8 \%, 77 \%$, and $56.9 \%$, respectively. A total of 805 Ixodes ricinus ticks were collected at the same time from both cattle (feeding ticks) and grazed pastures (questing ticks). The infection level of ticks, assessed by PCR assay, for Babesia
\end{abstract}

L. Lempereur $\cdot$ Y. Caron · B. Losson

Laboratory of Parasitology and Parasitic Diseases,

Department of Infectious and Parasitic Diseases,

Faculty of Veterinary Medicine, University of Liège,

4000 Liège, Belgium

M. Lebrun · P. Cuvelier · G. Sépult

Laboratory Animal Health Department of the Regional

Association of Animal Health and Identification (ARSIA),

5590 Ciney, Belgium

C. Saegerman

Research Unit in Epidemiology and Risk Analysis Applied to the Veterinary Sciences (UREAR), Department of Infectious and Parasitic Diseases, Faculty of Veterinary Medicine, University of Liège,

4000 Liège, Belgium

\section{Lempereur $(\varangle) \cdot$ B. Shiels}

Institute of Infection, Immunity \& Inflammation, College of Medical, Veterinary \& Life Sciences, University of Glasgow,

Henry Wellcome Building, 464, Bearsden Road, Glasgow G61 1QH Scotland, UK

e-mail: 1.lempereur.1@research.gla.ac.uk spp. DNA was $14.6 \%$ and $7.9 \%$ in feeding and questing ticks, respectively, whereas $21.7 \%$ and $3 \%$ of feeding and questing ticks were found be positive for A. phagocytophilum cDNA. Fifty-five PCR-positive samples were identified by sequencing as Babesia sp. EU1, of which five from feeding ticks were positive for both A. phagocytophilum and Babesia sp. EU1. The high density of wild cervids in the study area could explain these observations, as deer are considered to be the main hosts for adults of I. ricinus. However, the absence of Babesia divergens both in feeding and questing ticks is surprising, as the study area is known to be endemic for cattle babesiosis. Increasing cervid populations and comorbidity could play an import role in the epidemiology of these tickborne diseases.

\section{Introduction}

Anaplasmosis and babesiosis are major tick-borne diseases with a high economic impact on livestock productivity over much of the world. Both diseases are also a public health concern and are caused by intraerythrocytic protozoa of the genus Babesia spp. and bacteria within the genus Anaplasma, respectively. Ixodes ricinus, the most prevalent tick species in northern and western Europe, is an important vector of both these pathogens (Baumgarten et al. 1999; Becker et al. 2009; Bonnet et al. 2009).

In Europe, cattle are known to be the host of Babesia divergens, the etiological agent of red water. $B$. divergens has also been found in humans and wild ruminants, roe (Capreolus capreolus), and red deer (Cervus elaphus), Duh et al. 2005; Zintl et al. 2011), although the designation of red deer as a host is still under discussion. $B$. divergens is endemic in the southern part of Belgium (Losson and Lefevre 1989) but is considered absent from other parts of the country 
(Saegerman et al. 2007). To date, as far as we are aware, human babesiosis has never been reported in Belgium.

The availability of new molecular tools has considerably increased knowledge on the epidemiology and taxonomy of Babesia spp. A novel species recently described in Europe is Babesia sp. EU1, proposed as Babesia venatorum (Herwaldt et al. 2003). Roe deer are thought to be the natural host of this potentially zoonotic species. Babesia sp. EU1 is closely related to Babesia odocoilei (a parasite of white-tailed deer) and based on a 31-nucleotide difference over the complete 18S rRNA gene sequence has been placed phylogenetically in a sister group with $B$. divergens (Herwaldt et al. 2003). Detection of Babesia sp. EU1 has been recorded recently in Belgium (Lempereur et al. 2011). Anaplasma phagocytophilum is a polymorphic gramnegative bacterium that strictly parasitizes mammals; wild and domestic animals are the main reservoirs (Halos et al. 2010), but infection is potentially zoonotic causing human granulocytic anaplasmosis (HGA). The symptoms of this disease vary from asymptomatic infection to severe disease by way of nonspecific flu-like clinical signs (Bakken and Dumler 2008; Thomas et al. 2009). A. phagocytophilum causes hematologic changes characterized by bacteremia, leukopenia, and thrombocytopenia. The loss of white blood cells promotes suppression of the immune response and causes infected humans or animals to be more susceptible to other infectious pathogens (Woldehiwet 2006).

In cattle, A. phagocytophilum cause bovine granulocytic anaplasmosis (BGA), also called tick-borne fever (TBF), (Woldehiwet 2006). The symptoms associated with BGA are fever, a drop in milk production, joint swelling and abortion. In addition, many asymptomatic cases of infection are suspected (Pusterla and Braun 1997). In Europe, the majority of clinical cases are observed in June and September, when temperature and humidity are optimal for ticks and cattle are grazing tick-infested pasture (Pusterla et al. 1998). In southern Belgium, the first case was detected in 2005 (Guyot et al. 2011). BGA is thus regarded as an emergent disease in southern Belgium.

Because of numerous suspected cases of bovine anaplasmosis and the proximity of some cattle herds to wildlife habitats, this study was performed to measure seroprevalences for A. phagocytophilum and Babesia spp. in blood samples of cattle from farms in southern Belgium. The prevalence of $A$. phagocytophilum and Babesia spp. infection was also assessed in both feeding and questing ticks collected from cattle and grazed pastures.

\section{Materials and methods}

Eight farms in southern Belgium with a known history of anaplasmosis and/or babesiosis and where cattle are likely to have contact with wildlife were selected (target sampling) (Fig. 1). Participation of the farms in the survey was voluntary.

\section{Serological study}

In each farm, ten females (Holstein, Belgian White Blue, or Montbeliard breed) greater than 1 year of age were selected at random. Whole blood was taken into a dry container tube during the spring (May-June), summer (July-August), and autumn (September) of 2010. Centrifugation was performed at 3,000 rpm for $5 \mathrm{~min}$ at room temperature, and collected sera were stored at $-20^{\circ} \mathrm{C}$ in $1-\mathrm{ml}$ aliquots, until further use.

Sera were analyzed using an indirect immunofluorescence antibody test (IFAT) developed for $B$. divergens according to Gray and Kaye (1991). A B. divergens strain originating from a clinical case in Cherain (province of Luxembourg, Belgium) was used as the antigenic source. A positive control serum was kindly provided by Prof Pfister (Ludwig Maximilians University of Munich). Sera were tested at a dilution of 1:40 and 1:80. A. phagocytophilum antibodies were also detected by IFAT, using an IFA detection kit (VMRD, Pullman, USA) at a 1:40 dilution.

Ticks collection and analysis

In the participating farms, ticks were collected monthly, between May and September 2010, both from cattle (feeding ticks) and from the environment (questing ticks). In two farms the animals were grazing on two distinct areas (Fig. 1). Ticks were trapped in grazed pastures by flagging with a 100 by $100-\mathrm{cm}$ flannel cloth, performed when conditions were sufficiently dry (Bram 1978). Farmers collected ticks from cattle using forceps, mainly during milking.

Ticks were preserved in ethanol $70 \%$ and morphologically identified up to stage and species level using a standard key for morphological identification (Arthur 1963). Sex and level of repletion were also recorded.

Tick DNA extraction was performed on adults and nymphs using proteinase $\mathrm{K}(20 \mathrm{mg} / \mathrm{ml})$ and the method of Boom et al. (1990). This method is based on lytic activity and nucleaseinactivating properties of proteinase $\mathrm{K}$ together with the nucleic acid-binding properties of silica particles following by an ethanol/acetone purification. To remove potential false-negative results due to polymerase chain reaction (PCR) inhibition and to validate the efficiency of the DNA extraction, an initial PCR test targeting the tick 16S rRNA gene was performed using $16 \mathrm{~S}+1$ and $16 \mathrm{~S}-2$ primers (Baumgarten et al. 1999). PCR-negative samples were subsequently diluted to try and remove any potential inhibition.

A Babesia spp. genus-specific PCR based on the $18 \mathrm{~S}$ rRNA gene was performed on validated DNA extracts, as 
Fig. 1 Map showing the selected farms. Farm 1 and farm 6 have two different exploitation sites. In red, Babesia sp. EU1 found in ticks collected from cattle and grazed pastures

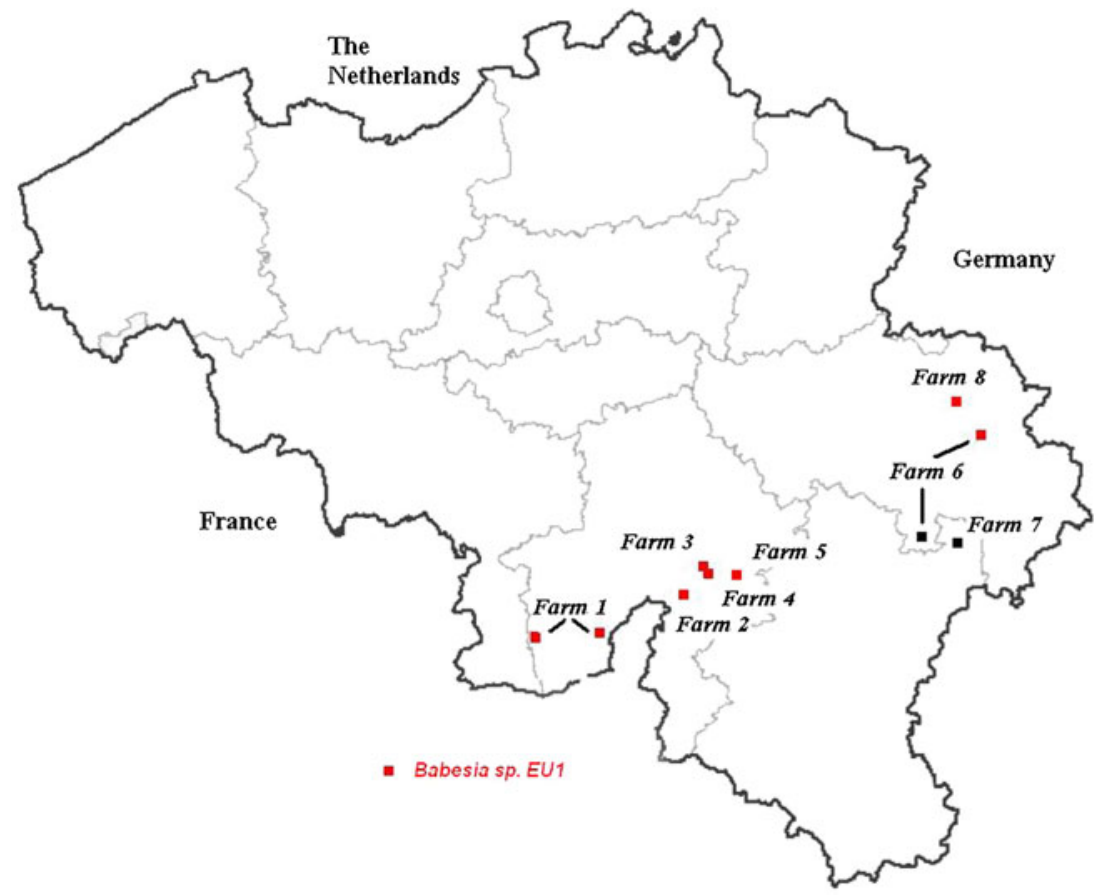

described by Casati et al. (2006) and adapted by Lempereur et al. (2011) Tick DNA samples positive for Babesia spp. were purified using the Qiaquick PCR purification Kit (Qiagen, Germany). Cycle sequencing reactions were then performed in both directions, using a BigDye terminator v3.1 (3730 DNA analyzer; Applied Biosystems) by Giga Genomics Facility (Liège University, Belgium) and by DBS Genomics (Durham University, UK). A consensus sequence representing each PCR amplicon was generated and sequence comparison performed using BLASTn searches in GenBank (www.ncbi.nlm.nih.gov) and alignment with Clustal W software (Thompson et al. 1994).

Detection of $A$. phagocytophilum cDNA was performed by RT-PCR (ABI 7000, Applied Biosystems) using Adiavet ${ }^{\circledR}$ Ana Pha Realtime kit (Adiagene, Saint Brieux,
France). This diagnostic kit can detect to a sensitivity of three biovars of $A$. phagocytophilum.

\section{Results}

Serology

Blood samples were collected in spring, summer, and autumn from 65 cows on seven different farms (Table 1). Unfortunately, field conditions during the summer prevented the collection of blood samples from 15 animals of the initial group of 80 selected for sampling.

For Babesia spp. seroprevalences of $10.7 \%, 20 \%$, and $12.3 \%$ were recorded in spring, summer, and autumn,
Table 1 Number of seropositive cows for Babesia spp. and $A$. phagocytophilum

\begin{tabular}{|c|c|c|c|c|c|c|}
\hline & \multicolumn{3}{|c|}{ Babesia spp. } & \multicolumn{3}{|c|}{ A. phagocytophilum } \\
\hline & Spring & Summer & Autumn & Spring & Summer & Autumn \\
\hline Farm 1 & 2 & 7 & 0 & 8 & 9 & 9 \\
\hline Farm 2 & 0 & 0 & 2 & 1 & 6 & 3 \\
\hline Farm 3 & 0 & 0 & 0 & 0 & 5 & 0 \\
\hline Farm 4 & 2 & 0 & 1 & 5 & 6 & 3 \\
\hline Farm 5 & 0 & 6 & 5 & 2 & 4 & 4 \\
\hline Farm 6 & 2 & 0 & 0 & 1 & 10 & 10 \\
\hline Farm 8 & 1 & 0 & 0 & 3 & 10 & 8 \\
\hline Total & 7 & 13 & 8 & 20 & 50 & 37 \\
\hline Percentage & 10.7 & 20 & 12.3 & 30.8 & 77 & 56.9 \\
\hline
\end{tabular}


respectively. Only one farm had no Babesia seropositive animals recorded throughout the experimental period. All farms had $A$. phagocytophilum-positive animals. The seroprevalences were $30.8 \%, 77 \%$, and $56.9 \%$ in spring, summer and autumn, respectively. Twelve (18.5\%) and 17 (26.2\%) cows were found to be either seronegative or seropositive throughout the complete period of the survey. Of the animals $55.3 \%$ presented a conversion in their serological status for A. phagocytophilum. Eleven cows $(16.9 \%)$ presented two seroconversions during the study period of 5 months, recorded as positive from the spring and autumn samples but then negative in the summer or only detected as seropositive in sample collected in the summer. Seventeen animals were positive before turn out to pasture in the spring; all of them had been grazed the previous year. Seventeen cows were found to be seropositive for both Babesia spp. and A. phagocytophilum during the same period of sampling.

\section{Ticks}

A total of 805 ticks were collected. All were identified as $I$. ricinus, both from cattle $(n=180)$ and pasture $(n=625)$, as shown in Table 2. The infection rate for Babesia spp. in PCR-validated tick samples was estimated to be $14.6 \%$ and $7.9 \%$ in attached and questing ticks, respectively, without any seasonal pattern recorded (or obvious). Fifty-five positive samples were identified as Babesia sp. EU1 after sequencing (accession no. JF922092-JF922099), on the basis of demonstrating $98 \%$ to $100 \%$ identity with the GenBank reference sequence (accession no. AY046575). Two Babesia-positive samples could not be identified to the species level. Babesia-positive samples were found in all seven of the sampled farms (Fig. 1).

The overall tick infection rate for A. phagocytophilum was $8 \%$, but strong differences were noticed according to the physiological status and stage of the ticks (feeding/questing; nymph/adult). Thus, $21.7 \%$ of the ticks collected from cattle were positive whereas only $3 \%$ of questing ticks gave a positive signal. However, almost all ticks collected from cattle were adults, whereas a majority of questing ticks were nymphs. Five female ticks collected from cattle were positive both for A. phagocytophilum and Babesia sp. EU1.

\section{Discussion}

Infection of cattle with the tick-borne pathogens, Anaplasma and Babesia, can have a serious impact on productivity, with the level of disease threat within a particular geographical region being strongly influenced by the species of these pathogens predominately transmitted by ticks. It is relevant, therefore, to have up-to-date information on the level of infection in cattle and the species of pathogens that are most

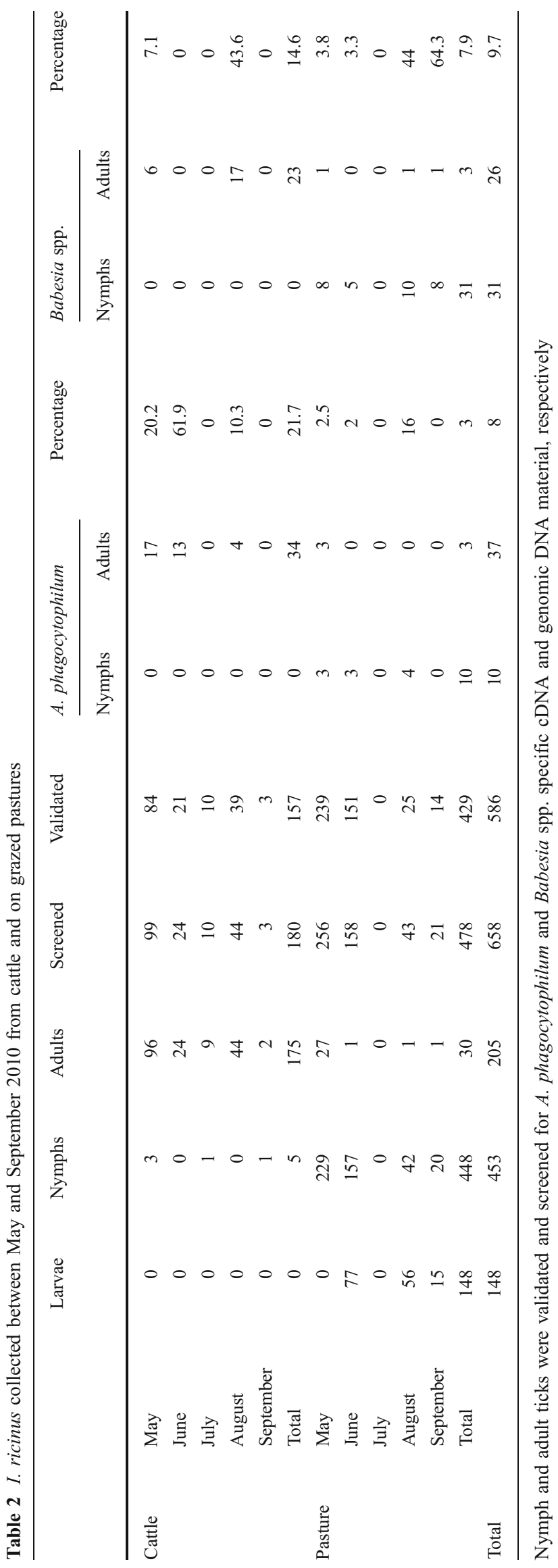


likely to be transmitted by ticks within regions under threat of disease. In the current study, the serological survey for Babesia spp. gave a prevalence $(14.3 \%)$, higher than the prevalence of $7.9 \%$ found in the same area by Losson and Lefevre (1989). The seroprevalence to A. phagocytophilum was also higher when compared to other previous European publications (Amusategui et al. 2006; Ebani et al. 2008). The fact that all the participating farms participated on voluntary basis had a confirmed history of tick-borne disease and were located in a known endemic area could explain the high seroprevalences due to targeted sampling.

The high seroprevalence against A. phagocytophilum was detected despite evidence that antibodies titer rapidly wanes, as indicated by double seroconversion results. This finding clearly has implications for detection of the true level of pathogen challenge that cattle encounter, and for both for diagnostic and epidemiological studies, the timing of sampling is of critical importance and should be performed close to the peak period of tick activity. Nevertheless, a significant proportion (26\%) of cattle were still positive before turn out to pasture suggesting that some cows remain as carriers throughout winter and may provide an overwintering reservoir population of $A$. phagocytophilum for feeding ticks in the spring.

BGA seems to be endemic in southern Belgium, as in a recent survey of 1,197 cattle, 320 tested positive for antibodies against $A$. phagocytophilum (ARSIA, unpublished data). This high level of infection and associated immunity could contribute to the relative lack of acute clinical cases of BGA in monitored herds. However, this may also be linked to $A$. phagocytophilum strain heterogeneity and vertebrate diversity (de la Fuente et al. 2005a; Granquist et al. 2010). Infection rates estimated for Babesia spp. in ticks were higher than generally expected for this region (Lempereur et al. 2011). This could well be due to targeted sampling, as mentioned above. However the high density of cervid populations in the study area may also have played a role. The infection rate found in ticks collected from cattle was also higher than that estimated for unfed questing ticks. It should be borne in mind that the ticks collected from cattle were all adult ticks, while the majority of those collected from the vegetation were nymphs. While the infection rates of both adult and nymph ticks will be influenced by the level of Babesia infection in the mammalian host, the known amplification of parasite in the tick salivary gland following a blood meal may increase the chances of detection by the PCR test. Furthermore, the adult population will be comprised of ticks that are transmitting and acquiring infection from a definitive host for Babesia, while the infection level in the nymph population will be predominately derived from adult female ticks (Zintl et al. 2003) that could, theoretically, have fed on a range of vertebrate hosts.

Babesia sp. EU1 was the only species found in both feeding and questing I. ricinus, despite the high number of ticks that were screened and the selection of farms located in a region previously identified as endemic for $B$. divergens. The same finding was previously described by Becker et al. (2009), who found no evidence for $B$. divergens sporozoites in engorged adult or unfed I. ricinus ticks. Cattle and deer are the main hosts of adult $I$. ricinus ticks and are potential carriers of $B$. divergens. One possible explanation for our results could be that the main stage of $I$. ricinus transmitting $B$. divergens in the study area is the larvae after transovarial transmission. This, possibility would need further investigation, as the accepted major transmission route is via feeding adults following transovarian transmission.

As deer can probably be a reservoir host for $B$. divergens (Zintl et al. 2011), it may be that cattle play the same role for Babesia sp. EU1 in natural habitats characterized by deciduous forests with large populations of wild ruminants. This hypothesis has yet to proven, indeed experimental validation of cattle acting as a reservoir for a Babesia species of deer has only been tested on Babesia capreoli (Adam et al. 1976; Gray et al. 1990; Malandrin et al. 2010). If it can be validated that cattle can act as a reservoir for Babesia sp. EU1, it would raise implications for the epidemiology and control of this zoonotic parasite. Wild cervids appear to act as a reservoir host for A. phagocytophilum (Rosef et al. 2009) as do probably cattle (de la Fuente et al. 2005b; Halos et al. 2010).

Since 1975, wild ruminant populations have doubled in southern Belgium, despite an increase in hunting activity (SPW-DGO3 Agriculture-Ressources naturelles et Environnement-Département de la Nature et des Forêts 2011). Expanding populations of wild cervids that act as important hosts for maintaining tick populations and acting as a reservoir for zoonotic tick-borne pathogens have the potential to alter the balance between the parasite and additional, potentially human, hosts. Therefore, knowledge of common animal reservoirs for transmission of zoonotic Babesia sp. will be useful for modelling the risk potential of this infection in humans: the results of this study implicate cattle as well as cervids.

The high seroprevalence to A. phagocytophilum in cattle suggest that this pathogen is endemic in southern Belgium. It would be expected, therefore, that ticks and mammalian hosts would be coinfected with A. phagocytophilum and additional tick-borne pathogens. Indeed, 17 cattle and five ticks demonstrated evidence of coinfection. It is known that coinfection of livestock, synchronous or not, with TBD can exacerbate disease morbidity and reduce host fitness (Zintl et al. 2003). Furthermore recent studies have provided evidence that interaction between tick-borne pathogen populations can influence TBD epidemiology. This can be manifest in a positive or negative manner and alter pathogen prevalence, pathogen transmission, and host susceptibility (Ginsberg 2008; Telfer et al. 2010). Therefore, to gain fuller insight 
into the factors that influence risk from tick-borne disease, future studies should attempt to capture data on interacting parasite communities rather than individual parasite species.

Acknowledgments Laetitia Lempereur is an early-stage researcher supported by the POSTICK ITN (postgraduate training network for capacity building to control ticks and tick-borne diseases) within the FP7PEOPLE-ITN program (EU Grant No. 238511). The authors are very grateful to the veterinarians and farmers involved in this survey for the sample collections. The authors would like to thank Dr. Christian Quinet and Ir Cédric Mullender for their scientific support and their supervision and Françoise Maréchal for her technical support.

\section{References}

Adam KM, Blewett DA, Brocklesby DW, Sharman GA (1976) The isolation and characterization of a Babesia from red deer (Cervus elaphus). Parasitology 73:1-11

Amusategui I, Sainz A, Tesouro MA (2006) Serological evaluation of Anaplasma phagocytophilum infection in livestock in northwestern Spain. Ann N Y Acad Sci 1078:487-490

Arthur DR (1963) British ticks. Butterworth, London

Bakken JS, Dumler S (2008) Human granulocytic anaplasmosis. Infect Dis Clin North Am 22:433-448, viii

Baumgarten BU, Rollinghoff M, Bogdan C (1999) Prevalence of Borrelia burgdorferi and granulocytic and monocytic ehrlichiae in Ixodes ricinus ticks from southern Germany. J Clin Microbiol 37:3448-3451

Becker CA, Bouju-Albert A, Jouglin M, Chauvin A, Malandrin L (2009) Natural transmission of zoonotic Babesia spp. by Ixodes ricinus ticks. Emerg Infect Dis 15:320-322

Bonnet S, Brisseau N, Hermouet A, Jouglin M, Chauvin A (2009) Experimental in vitro transmission of Babesia sp. (EU1) by Ixodes ricinus. Vet Res 40:21

Boom R, Sol CJ, Salimans MM, Jansen CL, Wertheim-van Dillen PM, van der Noordaa J (1990) Rapid and simple method for purification of nucleic acids. J Clin Microbiol 28:495-503

Bram RA (1978) Surveillance and collection of arthropods of veterinary importance. Agric Handb 518:108-109

Casati S, Sager H, Gern L, Piffaretti JC (2006) Presence of potentially pathogenic Babesia sp. for human in Ixodes ricinus in Switzerland. Ann Agric Environ Med 13:65-70

de la Fuente J, Massung RF, Wong SJ, Chu FK, Lutz H, Meli M, von Loewenich FD, Grzeszczuk A, Torina A, Caracappa S, Mangold AJ, Naranjo V, Stuen S, Kocan KM (2005a) Sequence analysis of the msp 4 gene of Anaplasma phagocytophilum strains. J Clin Microbiol 43:1309-1317

de la Fuente J, Naranjo V, Ruiz-Fons F, Hofle U, Fernandez De Mera IG, Villanua D, Almazan C, Torina A, Caracappa S, Kocan KM, Gortazar $\mathrm{C}(2005 \mathrm{~b})$ Potential vertebrate reservoir hosts and invertebrate vectors of Anaplasma marginale and A. phagocytophilum in central Spain. Vector Borne Zoonotic Dis 5:390-401

Duh D, Petrovec M, Bidovec A, Avsic-Zupanc T (2005) Cervids as Babesiae hosts, Slovenia. Emerg Infect Dis 11:1121-1123

Ebani V, Cerri D, Fratini F, Ampola M, Andreani E (2008) Seroprevalence of Anaplasma phagocytophilum in domestic and wild animals from central Italy. New Microbiol 31:371-375

Ginsberg HS (2008) Potential effects of mixed infections in ticks on transmission dynamics of pathogens: comparative analysis of published records. Exp Appl Acarol 46:29-41

Granquist EG, Bardsen K, Bergstrom K, Stuen S (2010) Variant and individual dependent nature of persistent Anaplasma phagocytophilum infection. Acta Vet Scand 52:25
Gray JS, Kaye B (1991) Studies on the use of gerbil-derived Babesia divergens antigen for diagnosis of bovine babesiosis. Vet Parasitol 39:215-224

Gray JS, Murphy TM, Taylor SM, Blewett DA, Harrington R (1990) Comparative morphological and cross transmission studies with bovine and deer babesias in Ireland. Prev Vet Med 9:193

Guyot H, Ramery E, O'Gradyc L, Sandersen C, Rollin F (2011) Emergence of bovine ehrlichiosis in Belgian cattle herds. Ticks Tick Borne Dis 2:116-118

Halos L, Bord S, Cotte V, Gasqui P, Abrial D, Barnouin J, Boulouis HJ, Vayssier-Taussat M, Vourc'h G (2010) Ecological factors characterizing the prevalence of bacterial tick-borne pathogens in Ixodes ricinus ticks in pastures and woodlands. Appl Environ Microbiol 76:4413-4420

Herwaldt BL, Caccio S, Gherlinzoni F, Aspock H, Slemenda SB, Piccaluga P, Martinelli G, Edelhofer R, Hollenstein U, Poletti G, Pampiglione S, Loschenberger K, Tura S, Pieniazek NJ (2003) Molecular characterization of a non-Babesia divergens organism causing zoonotic babesiosis in Europe. Emerg Infect Dis 9:942948

Lempereur L, De Cat A, Caron Y, Madder M, Claerebout E, Saegerman C, Losson B (2011) First molecular evidence of potentially zoonotic Babesia microti and Babesia sp. EU1 in Ixodes ricinus ticks in Belgium. Vector Borne Zoonotic Dis 11:125-130

Losson B, Lefevre F (1989) La babesiose bovine en Belgique. Une enquête sérologique en zone d'endémie. Ann Med Vet 133:421-426

Malandrin L, Jouglin M, Sun Y, Brisseau N, Chauvin A (2010) Redescription of Babesia capreoli (Enigk and Friedhoff, 1962) from roe deer (Capreolus capreolus): isolation, cultivation, host specificity, molecular characterisation and differentiation from Babesia divergens. Int J Parasitol 40:277-284

Pusterla N, Braun U (1997) Clinical findings in cows after experimental infection with Ehrlichia phagocytophila. Zentralbl Veterinarmed A 44:385-390

Pusterla N, Pusterla JB, Braun U, Lutz H (1998) Serological, hematologic, and PCR studies of cattle in an area of Switzerland in which tick-borne fever (caused by Ehrlichia phagocytophila) is endemic. Clin Diagn Lab Immunol 5:325-327

Rosef O, Paulauskas A, Radzijevskaja J (2009) Prevalence of Borrelia burgdorferi sensu lato and Anaplasma phagocytophilum in questing Ixodes ricinus ticks in relation to the density of wild cervids. Acta Vet Scand 51:47

Saegerman C, Claerebout E, Kalume M, Losson B (2007) Bovine babesiosis in Belgium: preliminary results of postal survey of veterinarians in 2006. Renc Rech Rumin 14:220

SPW-DGO3 Agriculture-Ressources naturelles et EnvironnementDépartement de la Nature et des Forêts, F.c. 2011. Report of Wild ruminants evolution in Belgium

Telfer S, Lambin X, Birtles R, Beldomenico P, Burthe S, Paterson S, Begon M (2010) Species interactions in a parasite community drive infection risk in a wildlife population. Science 330:243-246

Thomas RJ, Dumler JS, Carlyon JA (2009) Current management of human granulocytic anaplasmosis, human monocytic ehrlichiosis and Ehrlichia ewingii ehrlichiosis. Expert Rev Anti Infect Ther 7:709-722

Thompson JD, Higgins DG, Gibson TJ (1994) CLUSTAL W: improving the sensitivity of progressive multiple sequence alignment through sequence weighting, position-specific gap penalties and weight matrix choice. Nucleic Acids Res 22:4673-4680

Woldehiwet Z (2006) Anaplasma phagocytophilum in ruminants in Europe. Ann N Y Acad Sci 1078:446-460

Zintl A, Mulcahy G, Skerrett HE, Taylor SM, Gray JS (2003) Babesia divergens, a bovine blood parasite of veterinary and zoonotic importance. Clin Microbiol Rev 16:622-636

Zintl A, Finnerty EJ, Murphy TM, de Waal T, Gray JS (2011) Babesias of red deer (Cervus elaphus) in Ireland. Vet Res 42:7 